\title{
Privacy Preserving model for patient centric health record management using chaincode
}

vinodhini mani ( $\nabla$ vino.sha200744@gmail.com )

SRM Institute of Science and Technology https://orcid.org/0000-0001-8423-4768

\section{Prakash M}

SRM Institute of Science and Technology

\section{Research Article}

Keywords: IPFS, Health Records, Blockchain, Privacy, Security, Interoperability, Scalability

Posted Date: October 8th, 2021

DOl: https://doi.org/10.21203/rs.3.rs-942249/v1

License: (c) (i) This work is licensed under a Creative Commons Attribution 4.0 International License. Read Full License

Version of Record: A version of this preprint was published at Electronics on November 30th, 2021. See the published version at https://doi.org/10.3390/electronics10233003. 


\section{Abstract}

Cloud computing poses a challenge to the healthcare infrastructure, as it affects privacy, confidentiality, and security rules concerning large binary objects such as x-rays and CT scan reports. However, health records are stored and accessed using an encryption hash which is stored in the interplanetary file system (IPFS), called a peer-to-peer system. But the patient's data is sold, share for research purposes by their healthcare providers without their knowledge as it affects their privacy and security. In the healthcare industry today, customers face the issue of health record that lacks interoperability, resulting in difficulty aggregating and examining patient data. The objective of this research is to develop cybersecurity measurement approaches that ensure patient information security by protecting against cyber threats using blockchain technology based on healthcare IT. Consequently, this paper proposes an innovative solution to the problem, namely Patient-centric healthcare data management (PCHDM). It was built using IPFS, a permissioned distributed ledger system that uses Hyperledger Fabric, which stores health records, but only with the permission of the owner. A unique cryptographic public key encryption algorithm is used to encrypt IPFS data to build an electronic health record blockchain system. Our platform offers two types of solutions: (i) a solution that utilizes a database of hyper ledger fabric, which is an on-chain database, (ii) off-chain solutions which encrypt data and store it securely off-chain using IPFS. A robust blockchain solution for PCHDM will be created by encrypting the data stored in IPFS using appropriate public key cryptographic algorithms. To determine which blocks should be incorporated into the blockchain, the Byzantine Fault Tolerance is applied in the health chain architectural model. This system hosts smart contracts and application logic as well as smart contracts known as "chain code" via container technology. As part of this research, health record hashes were stored on the blockchain and the actual health data was stored off-chain in IPFS, which is the decentralized cloud storage system that achieves scalability. Due to the encryption of healthcare records with a hash, this model proves that unauthorized access is impossible because the records are more scalable, interoperable, and reliable. Stakeholders are more confident in collaborating and sharing their medical records with this model.

\section{Introduction}

Blockchains are databases that store time stamps on the records in a cryptographically secured chain of blocks. Despite their ease of use and reliability, traditional EHR systems present numerous privacy and security challenges. ${ }^{[1]}$ The health record such as electronic medical records (EMRs), electronic health records (EHRs), and personal health records (PHRs) clinical pictures and patient data, for example, doctor name, individual measurements, home checking gadget information, what's more, different information handled by specialists in a content organization. As hospitals process such vast volumes of image data, they will need more storage space, and access to such data will rise rapidly. Consequently, medical images must be transmitted and stored securely without interruption. Data centers that currently store and transfer medical images and patient information are deemed insecure, inaccessible, and have concerns about privacy and security. Throughout the past two decades, medical image processing companies have experienced additional challenges caused by record breach episodes within large 
medical data centers ${ }^{[2]}$ A patient-centric system, therefore, should allow patients online access to their medical images and a central infrastructure, while also allowing them to log in to their account anytime. As a consequence of evolving towards decentralized technology, such as blockchain, ${ }^{[3]}$ a potential solution to reducing these barriers has emerged that may support widespread adoption for a patientcentric system. The size of medical images and records poses a significant problem for their storage on a blockchain. No blockchain can provide medical images of orders of magnitude greater than those necessary for public use ${ }^{[4]}$. The decentralized storage problem is solved by implementing the Interplanetary File System (IPFS) was created by Protocol Labs ${ }^{[5]}$. Hypermedia can be shared and stored in IPFS which is a peer-to-peer (P2P) and content-addressable file system. Because IPFS offers off-chain storage, IPFS is compatible with any blockchain network. It is important, however, to avoid unauthorized access to medical images when using distributed storage solutions, as well as concerns regarding patient privacy. Over the past two decades, the practice of registering and sharing medical health records has changed significantly, largely due to stricter standards, the use of sophisticated technology, and curated diagnoses and treatments. Medical images, usually transferred via CDs or DVDs, are exchanged by patients, physicians, and hospitals to help with diagnosis. However, complications like patient and lab reporter mistakes can result in damaged images ${ }^{[6]}$. In the early days of blockchain technology, MedRec [7] became the first suggestion for an electronic patient record management system that was implemented by blockchain technology. Ethereum Blockchain and smart contracts store detailed accessibility data. Blockchain does not store the medical records, but rather the third-party database operated by healthcare providers. Infringement or misuse of these records is therefore still possible. In Blockchain, the Healthcare management systems ${ }^{[8]}$ patient's private keys are encrypted by recording data on the blockchain. In order to decrypt data, hospitals and researchers obtain consent from a patient's public key to decoy the data. This contrasts dramatically with our approach, in which patients are the only ones who have access to their data. As part of the current consortium blockchain initiative, Zhang et al. ${ }^{[9]}$ proposed a scheme for the sharing of privacy-protecting data for personal health information (PHI). This scheme created two kinds of blockchain by using the Merkle DAG structure and some consensus mechanisms. By combining public-key encryption with keyword searches, a PHI sharing protocol is proposed based on blockchain for secure data protection, privacy protection, access control, and secure search. Although the scheme enables data access deadlines, data revocation, and so on, it does not allow full control. Researchers Wu et al. developed a traceable encryption system that ensures accuracy, non-repudiation, and privacy within the blockchain [10]. Researchers have tackled the issue of sharing secret keys with entities that do not contain specific information about the user. In the case of a tampered or abused shared key, analyzing the source of secret keys becomes difficult. Access control systems are also slow due to leaks of confidential information. As a privacy barrier, the authors incorporated attribute-based encryption algorithms in order to protect the secret keys. Decryption is not guaranteed to be secure, however. In order to access addresses stored on IPFS, it needs to be authenticated by multiple parties. Data Owners (DO) share their data by uploading and accepting the requirements imposed by the Multi-Party Authority (MPA) and registering the data address (hash). By encrypting the data with a symmetric key standard, and sending it to IPFS with the others, along with their other keys encrypted by MPA and DO's shared private 
key, this can be accomplished. Smart contracts are used to manage reputations. During the process of transferring data from one individual to another, the proposed system requires multiple cycles of encryption and decryption, thereby increasing operating costs. The inefficiency of this method occurs because every new user has to re-encrypt their data every time. A centralized server also encrypts the data, which is frequently untrustworthy. An electronic health record (EHR) access control policy is presented in another study conducted in ${ }^{[9]}$. At first, symmetric keys are used to encrypt the data that needs to be shared. It assists the sender and receiver in fetching and sending data by acting as a mediator. To re-encrypt a key that is sent to the proxy, the owner shares the private key with the recipient. Data is decrypted with the private keys by the proxy and the new key is used to re-encrypt the files. The proxy then pushes the encrypted files to the receiver who decrypts them. Researchers ${ }^{[11]}$ reported that PACS access was compromised and medical images were permanently modified due to default accounts, cross-site scripting, and web server vulnerabilities. There are serious privacy concerns associated with accessing and sharing patient information, such as storing identifying information in a central repository, limiting the ownership of images, and mismatching information between patient registries and health databases ${ }^{[12]}$. Therefore, it is important that the underlying architecture applies to a decentralized architecture and that privacy configurations, data provenance, security protocols, authentication, and electronic consent be identified. Several researchers have been developing frameworks that combine cloud computing and a blockchain to share medical health records ${ }^{[13]}$. An individualized dermatologyspecific blockchain-based treatment management system is presented in [13]. Medical records may be shared selectively by patients using a private digital key. A machine learning-based analysis algorithm could use different images stored in a blockchain network. However, cost-effectiveness and scalability must be considered before standardizing this approach.

Hence there is a need of technology that gives the effective storage of data. To achieve this an effective patient centric distributed architecture to for storing of patient centric data and simultaneously concern about parameters like Privacy, Security, Integrity, Interoperability and scalability. This paper contributes the following to the discussion:

This paper contributes the following to the discussion:

In this research, we are developing a PCHDM that ensures the integrity, security, scalability, and privacy of health records while providing patients with complete control. Health information is mainly stored on the blockchain as hashes, whereas the original vast quantities of data are maintained off-chain in IPFS, to ensure scalability and efficiency. The research uses PCHDMAC-SC, where PCHDMAC-SC controls access conditions for registered stakeholder groups and does not use any form of incentive mining beyond equitable access to the system. The temporary view called patient centric view of the health record is created and after the patient grant the record is successfully stored in the IPFS system. Hence this system protects the privacy of the patient using role-based access control and their scalability and interoperability are tested and evaluated using testcases. The performance evaluation results show our proposed system has improves scalability and interoperability compared with the existing system. 


\section{Materials And Methods}

\subsection{Components of PCHDM}

\subsubsection{Blockchain}

A smart contract platform (SC) platform was incorporated into the Hyperledger system. SC stores rules relating to contract negotiations. For medical information sharing efficiently and reliably without having to rely on a single authority, we are employing Hyperledger Fabric, a permissioned blockchain based on pre-specified parties. Hyperledger Fabric offers the advantage of employing the Byzantine fault tolerance consensus protocol without requiring mining or an associated currency as a means of achieving consensus. Hyperledger blockchain uses a Merkle Directed Acyclic Graph tree structure as its state database, which can be replicated using IPFS objects. Therefore, IPFS can be used to model an off-chain and on-chain blockchain for health record storage. By implementing the PCHDMAC-SC protocol, we created a transparent, controlled access system that prevented hacking without patient consent using an Hyperledger blockchain.

\subsubsection{Distributed File system}

A cryptographic hash represents a unique fingerprint for each file within IPFS, a peer-to-peer (P2P) protocol. To make the contents immutable, the hash address is applied ${ }^{[14]}$. Merkle DAGs combine Merkle trees with DAGs in IPFS file storage. Rather than relying on location-based addressing, IPFS's key feature is that access to medical images can be accomplished through content-based addressing. Due to IPFS, bandwidth costs can be reduced, image download speeds can be enhanced, and a large volume of data can be distributed without duplication, which can save storage space. The hash value of an IPFS file cannot be changed so IPFS is an immutable storage mechanism.

\subsubsection{Services Provided to Members}

A cryptographic procedure that validates identity, generates and verifies signatures, and generates and verifies certificates, as well as verifying the identity of the user is described in ${ }^{[15]}$. This model uses FabricCertificate Authority (CA) as the interface for Services Provided to Members. A participating organization can, however, use an external CA.

\subsection{A Background of the PCHDM System}

An illustration of Health chain's proposed architecture can be seen in Figure 1. User requests are sent to the fabric network by the Dapp through an API called the composer, which is interactively handled by the Dapp admin. Through GET calls to the composer, the Angular framework can access the on-chain database and retrieve data based on the current state as returned by the API. Smart contract composer creates decentralized applications based on blockchain business networks. With Hyperledger Fabric ${ }^{[16]}$ network participants can validate medical data entries via smart contracts named chain codes. This 
technology was developed for distributed ledger solutions. Bitcoin [17] was created specifically for financial transactions, and Hyperledger is for storing health records.

Hyperledger Composer uses permissioned blockchain based on Hyperledger Fabric to develop web applications for single organizations using three peer nodes. Three peer nodes are used by the organization, one serving as a validating peer node, while another serves as an ordering node (Kafka) that is used to register participants. In this system, multiple peers to access the corresponding database, IPFS for distributed storage of data, a Solo Order Node, a Data Certificate Authority, a Membership Service Provider and smart contracts for blockchain connectivity. In order to verify the system's scalability, multiple peers can be added to multiple locations on different machines. Smart contractors have access to ledgers and have ledger access through this framework. Peer nodes are connected to the application, which then updates the ledger via smart. The three peer nodes in the organization are peernode0 (PE0), peernode1 (PE1), and peernode2 (PE2), each of which contains its own ledger and smart contract copies. In Hyperledger Composer, a single channel $(\mathrm{CH})$ facilitates communication with peers. This network generates a transaction T1 for our application APP1 and sends it to peernode0, peernode1, and peernode2. The chain codes are installed by the peers based on the execution of a transaction. When querying or altering the ledger, the application uses chain codes to interact with peers. $\mathrm{WL}_{\operatorname{tr}(r)}, \mathrm{WL}_{\mathrm{ph}(r) \text {, }}$ and the current transaction hash $W L_{h(r)}$ make up a block containing a patient's health record $r$ in the ledger. The block workload can be calculated using $\mathrm{WL}_{\mathrm{Tot}(\mathrm{r})}$

$W L_{T o t(r)}=W L_{t r(r)}+W L_{p h(r)}+W L_{h(r)}$

\section{Health Record Structure}

The health record consists of patient's profile, Diseases Diagnosed, Address Location, Medicine, Doctor Suggestion, Next Review Notes, Doctors Name, Hospital id, Scan and Test image reports.

\section{Owner of Record}

Patients own their medical records. A patient will have to create chain code PCHDMAC-SC on the Hyperledger blockchain and store it there. IPFS networks allow patients to define access rights to their health data. Each PCHDMAC-SC defines this within its own context.

\section{Data Uploader}

Physicians may upload their medical data to Data Uploaders. Adding encrypted clinical data of the affected person to the IPFS community and confirming the preliminary transaction at the blockchain are the high responsibilities of the data uploaders.

\section{Data Users}

All those interested in obtaining clinical or medical data about patients, whether they are physicians, hospitals, insurance companies, or researchers, are considered Data Customers. PCHDMAC-SC contains 
role-based access control mechanisms that defines how patients can grant access privileges to data users.

\subsection{Data Encryption}

The integrity and confidentiality of blockchain data is ensured by cryptographic techniques. Figure 2 outlines how patients and doctors interact when accessing health records. Bringing up the health records stored in the IPFS, the doctor requests permission to access it. As shown in Table1 and patients approve or grant requests from permissioned users based on their roles and rules-based access privileges. Rather than sharing all the information about the patient, it creates a patient-centric view of the records based on the request. Sk is used to access records in a definite session, and the patient-centric view is encrypted and stored in IPFS with the session key. Doctors and patients receive encrypted patient-centric views and encryption session key Sk. Doctors can decrypt Sk and patient-centric views for an update on the health record. The patient has been notified after the update of his record in IPFS. If the patient commits to his health record, then the Sk and the patient-centric view will automatically be deleted.

\subsection{PCHDMAC-SC}

In order to obtain access to the IPFS health record of the patient, the Doctor requests permission. Rolebased access control permissions enable the patient to approve or deny requests from authorized users as shown in Table 1. Users or Doctors can only access health records if their access control permissions permit them to write, read and update them. In this health chain framework, other stakeholders such as Pharmacists and Lab Managers can only access the patient-centric view of the health records for a specific session if their object ID matches the ownership ID.

Table 1 Role and Rule Based Access and Authentication

\begin{tabular}{|lll|}
\hline Role Name & Permission By Patient & Health Record \\
\hline Patient & Authorized & Read and write \\
\hline Doctor & Authorized & Read and write \\
\hline Pharmacist & Authorized & Patient Centric view Read \\
\hline Receptionist & Authorized & Patient Centric view Read \\
\hline Researcher & Authorized & Patient Centric view Read \\
\hline Insurance Agent & Authorized & Patient Centric view Read \\
\hline Referral Doctor & Authorized & Read and write in emergency \\
\hline Lab Technician & Authorized & Read and write \\
\hline
\end{tabular}

\subsection{PCHDM Algorithm}


The four participants in our framework are $\mathrm{Pa}, \mathrm{D}, \mathrm{Ph}$, and $\mathrm{LT}$, where $\mathrm{P}$ is the patient, $\mathrm{D}$ is the Doctor, $\mathrm{Ph}$ Pharmacist, and LT is the lab technician. Hyperledger-CA issues public key certificates to $n$ participants including patients, doctors, lab technicians, and pharmacists. A key pair will be generated for all the participants. Algorithm 1 explains that the patients Pan give grant access to Doctors $D_{n}$ to access their health record $\mathrm{HR}_{\mathrm{n}}$ based on PCHDMAC-SC. Hence the system generates a patient centric view $\mathrm{Pa}_{\mathrm{cvn}}$ of the health record $H R$. Based on the Doctor $D_{n}$ request, the attribute-based data is retrieved from the $\mathrm{Pa}_{\mathrm{cvn}}$ instead of sharing whole patient health records. In other words, the patient-centric view is a subset of the health record. Patient centric view data is encrypted using key value pairs, especially medical images that are encrypted using Steganography with the session keys. To update health, record the doctor should call the method update $\mathrm{UPa}_{\mathrm{cvn}}$. Then the patient must commit to the update. Then it added to IPFS storage permanently.

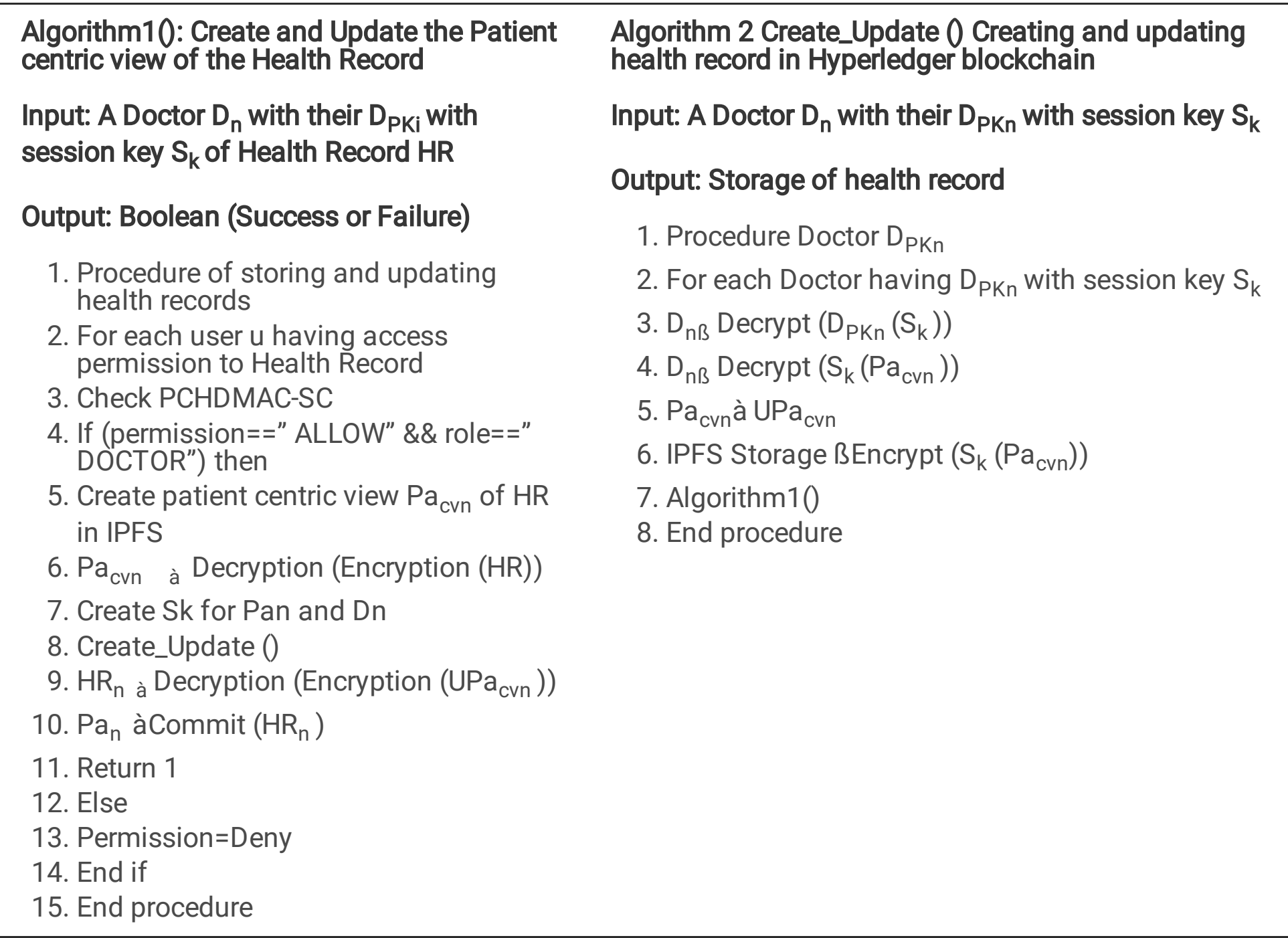

\subsection{Implementation of PCHDM Protocol}

The protocol has implemented as four steps

- Add Users

- Add Records 
- Assuring Authorized Users Have Access

- Records retrieval

\section{Results And Discussion}

An Intel ${ }^{\circledR}$ Core TM i7-3632 QM processor running at $2.20 \mathrm{GHz}$, Hyperledger composer, IPFS and memory $8 \mathrm{GBwas}$ used for the experiment. Every transaction in health chain makes use of smart contracts chain code [18], the IPFS storage system, and networking entities create the framework for the application. Health chain's user sign up allows physicians, pharmacists, receptionists, and other health care providers to register using their respective roles. Using their email address and password, the user can use their user type to sign in after registering as shown in the Figure3. Receptionists can accept or reject appointments booked by participants using patient IDs and update the participants based on the patient's information. Patient consults the doctor after the appointment has been approved by the receptionist, and the doctor creates the patient's medical record. IPFS allows doctor to upload medical notes or diagnosis results as shown in the Figure 4. The prototype has undergone a number of tests in order to validate its functionality and evaluate its performance. An assessment of the health chain framework systems is realized through the application of four case studies that illustrate efficiency, scalability, storage, and security. (i)Record storage that is efficient (ii)Security at a high level (iii)Privacy enhancements (iv)Enhanced data scalability. The above four test case are tested with different possibilities and their test results are recorded. In [19] the researcher has explained the process and consequences of recent healthcare management in blockchain. Table 2 shows the comparative analysis of existing and proposed patient centric health storage model, from this table our proposed model concentrate on scalability, privacy and security.

\section{Table 2 Comparison of proposed and existing model}

\begin{tabular}{|llllll|}
\hline Models & $\begin{array}{l}\text { Ease of } \\
\text { scaling }\end{array}$ & $\begin{array}{l}\text { Protection of } \\
\text { personal data }\end{array}$ & $\begin{array}{l}\text { Confidential } \\
\text { information }\end{array}$ & $\begin{array}{l}\text { Data } \\
\text { integrity }\end{array}$ & $\begin{array}{c}\text { data } \\
\text { security }\end{array}$ \\
\hline PCHDM & $\checkmark$ & $\checkmark$ & $\checkmark$ & $\checkmark$ & $\checkmark$ \\
\hline $\begin{array}{l}\text { Shen et al } \\
{[20]}\end{array}$ & X & X & $\checkmark$ & $\checkmark$ & $\checkmark$ \\
$\begin{array}{l}\text { Wang et al } \\
{[21]}\end{array}$ & $x$ & $x$ & $\checkmark$ & $\checkmark$ & $\checkmark$ \\
\hline
\end{tabular}

In this research, we used a dataset of variable sizes of images and text to test our assumptions. This first experiment employed a blockchain framework to calculate transaction latency as illustrated in Figure 5. The latency of a transaction reflects how long it takes to commit. It is a distributed parameter across nodes in the network. If there are $p$ number of nodes in the health chain network, $\mathrm{TR}_{\mathrm{Lan}}$ is the transaction latency, $T R_{\text {Ctp }}$ is the confirmation time in the network nodes and $t_{S e p}$ is the transaction submit time in seconds then. The latency of a transaction in the health chain network is $t$, the confirmation time $T R_{C t p}$ in the network nodes is $T R_{C t p}$, the time it takes for the transaction to be sent in seconds is $T R_{\text {Sep. }}$. 
$T R_{\text {Lan }}=T R_{\text {Ctp }}-T R_{\text {Sep }}$

The network ledger has been updated through the use of eight groups of transactions ranging from 5,15, $25,30,35,45,50$ and 55 as shown in the Figure 6 . In this configuration, the first five transactions across the network were committed in 104 seconds, and the final 55 transactions took 161 seconds on average. Thus, as the number and complexity of peers, and transactions increase, the longer it takes to execute a transaction. From the figure 7 it shows the time latency increases with increase of number of peer nodes. Transaction throughput is the number of transactions which valid and committed from the total executed transactions.

$T R_{\text {tpp }}=T R_{v c p} / T R_{\text {totalp }}$

The average upload and download time of storing the medical image data for the sample $100 \mathrm{mb}$ is 34 seconds and 43 seconds as shown in the figure 8 . Then the average upload and download time of the sample $100 \mathrm{mb}$ health record document file is 39.3 and 53.5 respectively as shown in the Figure 9 . When assets are successfully loaded and written to a database, the asset latency is measured. In a blockchain network with P nodes, AS_Lp represents the Asset Latency. The response time TR_Resp is measured in milliseconds and the asset submit time TR_AS_Subp is measured in milliseconds.

$$
\text { AS_Lp = TR_Resp- TR_AS_Subp }
$$

Figure 10 shows the asset time to commit in the blockchain form sample set of transactions. As a result, this system can process a large dataset with low latency. From the Figure [8,9] the average upload and download latency is maintained for all the transaction sets without any interrupt that shows the scalability of the system has improved using Hyperledger health record storage.

\section{Conclusion}

We have demonstrated the use of Hyperledger fabric and Hyperledger composer for secure electronically stored health records, and the use of a permissioned blockchain has store has data confidentially and securely. With the help of data encryption methods and role-based access control methods, a secure data storage architecture and streamlined access control between stakeholders is proposed. A proper health data network can be established by implementing and evaluating the proposed methodology with a few applications for health records. Considering all healthcare transactions has stored as hash encrypted data values on the blockchain as a result of prototype implementation and analysis, the approach is robust and non-tam parable. Various scenarios are simulated to evaluate performance. Additionally, this study explores the business processes and technology framework associated with blockchain. An easier and more efficient way to store, transfer, manage, and access electronic health records has been developed by introducing cryptographic technology. In order to handle increasing peer node numbers with higher efficiency, research must improve role-based access control mechanisms are required.

\section{Declarations}


Competing interests: The authors declare no competing interests.

\section{References}

1. Heart, T., Ben-Assuli, O.: Shabtai, I. A Review of PHR, EMR and EHR Integration: A More Personalized Healthcare and Public Health Policy. Health Policy Technol. 2017, 6, 20-25

2. Seh, A.H.; Zarour, M., Alenezi, M., Sarkar, A.K., Agrawal, A., Khan, A.R.. Healthcare Data Breaches: Insights and Implications. Healthcare 2020, 8, p. 133

3. Mettler, M.: Blockchain Technology in Healthcare: The Revolution Starts Here. In Proceedings of the IEEE 18th International Conference on e-Health Networking, Applications and Services (Healthcom), Munich, Germany, 14-16 September 2016; pp. 1-3

4. Halamka, J.D., Lippman, A.: The Potential for Blockchain to Transform Electronic Health Records. Harvard Bus. Rev. 3, 2-5 (2017). https://hbr.org/2017/03/the-potential-for-blockchain-to-transformelectronic-health-records Available online:; Ekblaw, A. (accessed on 27 December 2020)

5. Benet, J. IPFS-Content, Addressed, Versioned, P2P File System (DRAFT 3). 2014. Available online: https://arxiv.org/abs/1407.3 561 (accessed on 22 November 2020)

6. Erickson, B.: Experience with Importation of Electronic Images into the Medical Record from Physical Media. J. Digit. Imaging 2011, 24, 694-699

7. Azaria et al.," MedRec: Using Blockchain for Medical Data Access and Permission Management," Open and Big Data (OBD) International Conference on, pp. 25-30, 2016

8. Citius Tech," Blockchain for Healthcare," 2018. [online]. Available: https://www.citiustech.com/uploads/knowledgehub/pdf/blockchain-forhealthcare-341.pdf

9. Blockchain for Supply Chain. Accessed: Mar. 25, 2018. [Online]. Available: https://www.ibm.com/blockchain/supply-chain

10. Tith, D., Lee, J.-S., Suzuki, H., Wijesundara, W., Taira, N., Obi, T., and Ohyama, N., "Application of blockchain to maintaining patient records in electronic health record for enhanced privacy, scalability, and availability," Healthcare Inform. Res., vol. 26, no. 1, pp. 3-12, 2020.Appl. Sci. 2021, 11, 1964 of 20

11. Eichelberg, M., Kleber, K., Kämmerer, M.: Cybersecurity Challenges for PACS and Medical Imaging. J. Digit. Imaging 33, 1527-1542 (2020)

12. Vest, J.R., Gamn, L.D.: Health Information Exchange: Persistent Challenges and New Strategies. J. Am. Med. Inform. Assoc 17, 288-294 (2010)

13. Tung, J.: Nambudiri, V. Beyond Bitcoin: Potential Applications of Blockchain Technology in Dermatology. Br. J. Dermatol. 2018, 179, 1013-1014

14. Tiwari, A., Batra, U.. IPFS enabled blockchain for smart cities. Int. j. inf. tecnol. 13, 201-211 (2021). https://doi.org/10.1007/s41870-020-00568-9Dannen, C. (2017). Introducing Ethereum and Solidity. Springer 
15. Androulaki, E., Barger, A., Bortnikov, V., Cachin, C., Christidis, K.: De Caro, A., Enyeart, D., Ferris, C., Laventman, G., Manevich, Y., et al. (2018). Hyperledger fabric: a distributed operating system for permissioned blockchains. In Proceedings of the Thirteenth EuroSys Conference, page 30. ACM

16. Nakamoto, S. et al.: (2008). Bitcoin: A peer-to-peer electronic cash system

17. Foschini, L., Gavagna, A., Martuscelli, G., Montanari, R., "Hyperledger Fabric Blockchain: Chaincode Performance Analysis," ICC 2020-2020 IEEE International Conference on Communications (ICC), 2020, pp. 1-6, doi: 10.1109/ICC40277.2020.9149080.0

18. Kumar, Shivansh\& Bharti, Aman \& Amin, Ruhul.: (2021). Decentralized secure storage of medical records using Blockchain and IPFS: A comparative analysis with future directions. Security and Privacy. 10.1002/spy2.162

19. Shen, B., Guo, J., and Yang, Y.: Medchain: Efficient healthcare data sharing via blockchain. Applied Sciences 9(6), 1207 (2019) 0

20. Wang, H., Song, Y.: Secure cloud-based ehr system using attribute-based cryptosystem and blockchain. Journal of medical systems 42(8), 152 (2018) 0

\section{Figures}

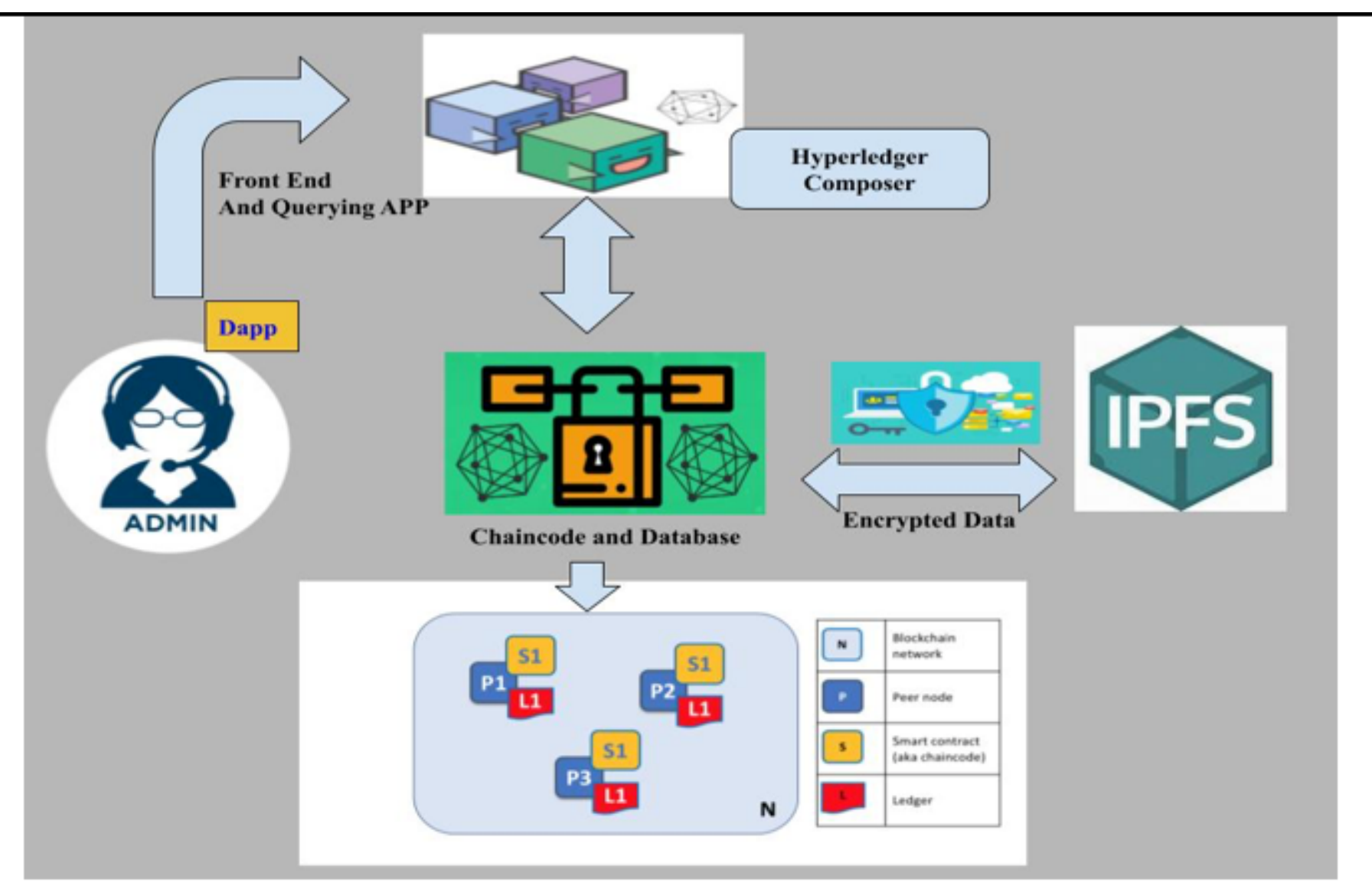

Figure 1 


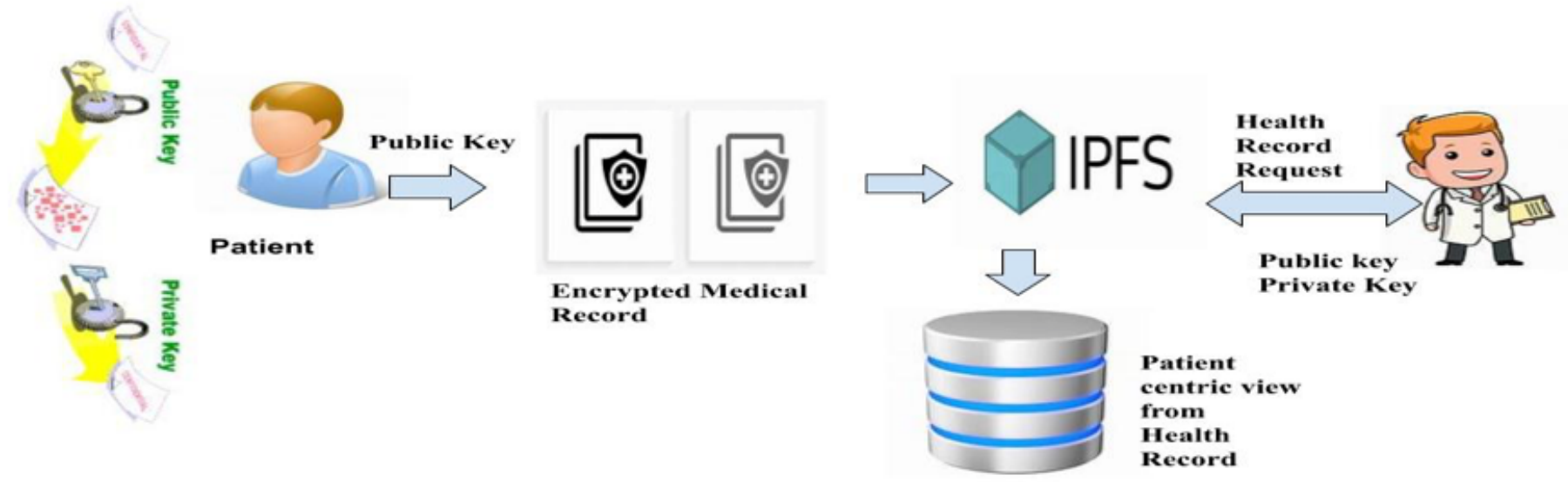

Figure 2

Health Record Creation and Updating 
Sign Up

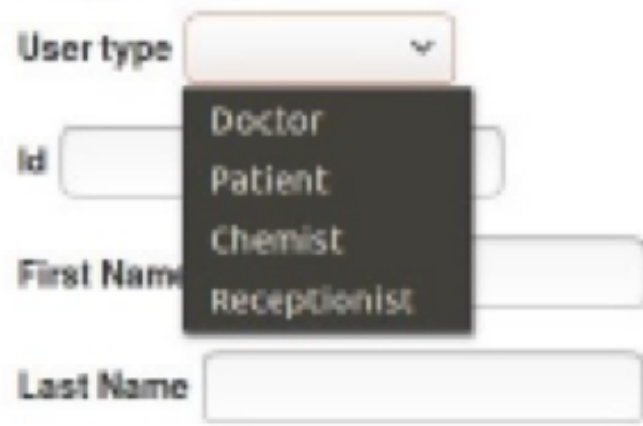

Email Address

Password

Age

Gender

Signup

\section{Login Form}

Not registered?

Sign Up

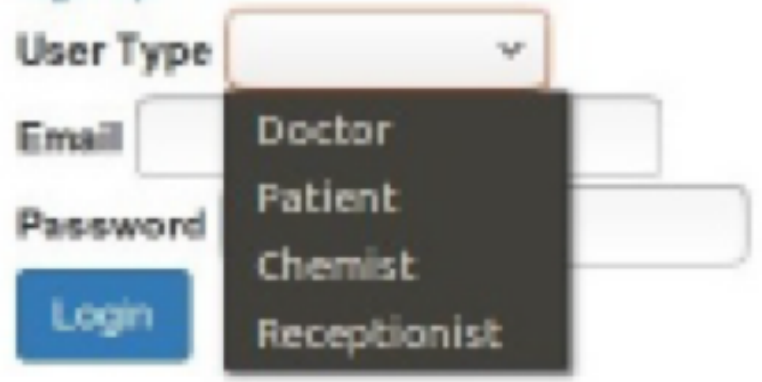

\section{Figure 3}

User Sign Up and Login Form 


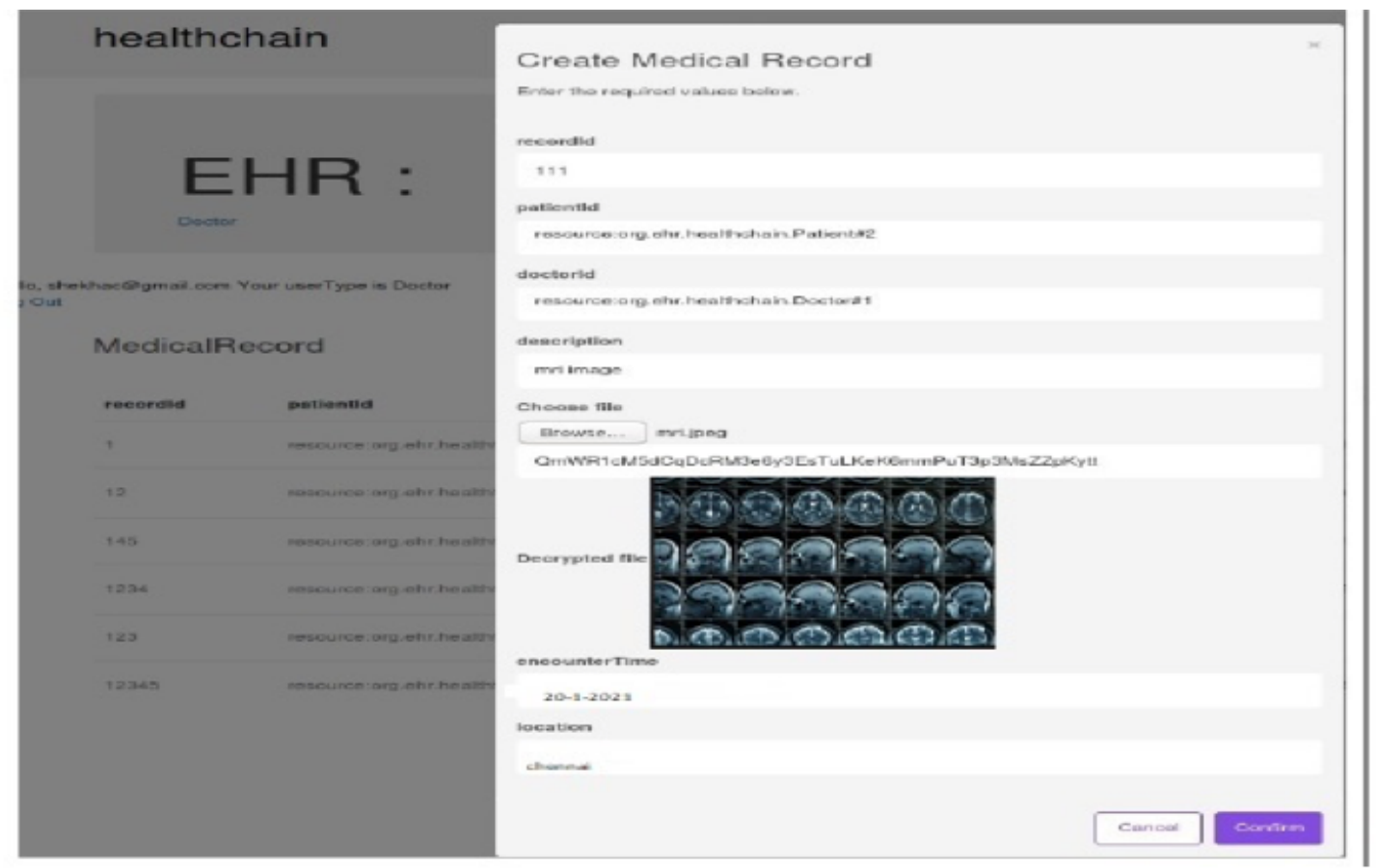

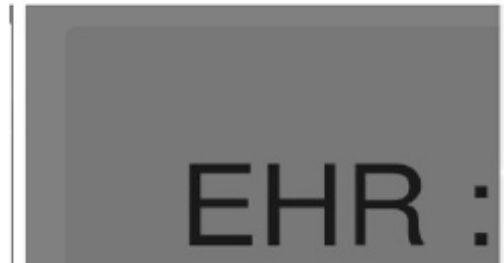

Patient

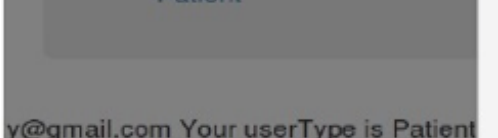

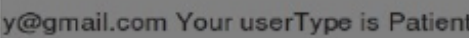

\section{AddOwnership}

ownershipld

11

22

33

\section{Create asset}

Enter the required values below.

ownershipld

m

\section{recordld}

resource:org.ehr.healthchain.MedicalRecord\# 111

userld

8

permissionType

O READ O WRITE O DENY

roleType

- DOCTOR PATIENT CHEMIST RECEPTIONIST

Cancel

Confirm

\section{Figure 4}

Creating Health Record of Patient 


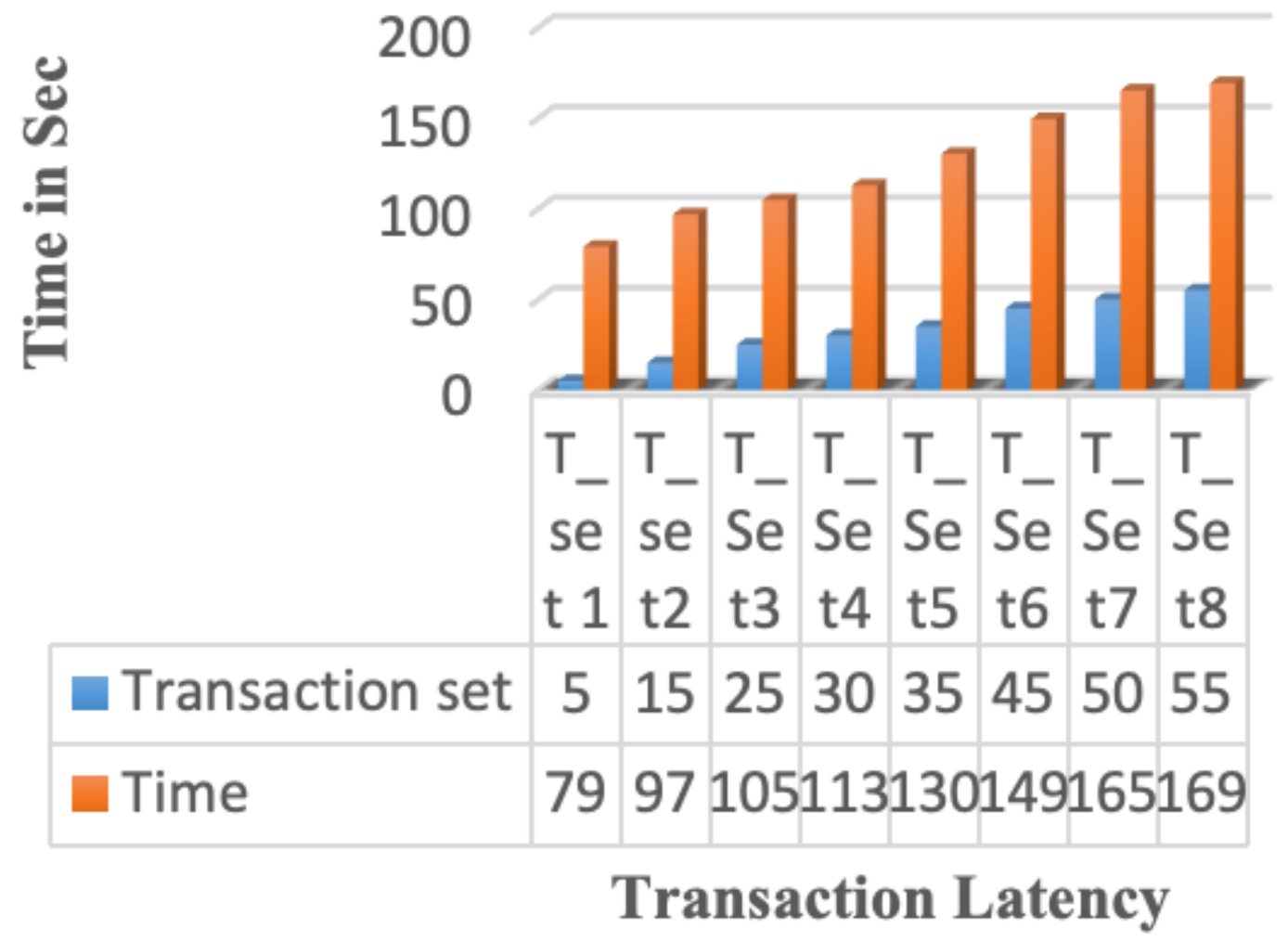

Figure 5

Latency comparison with organization peernode 1 for sample set of transactions 


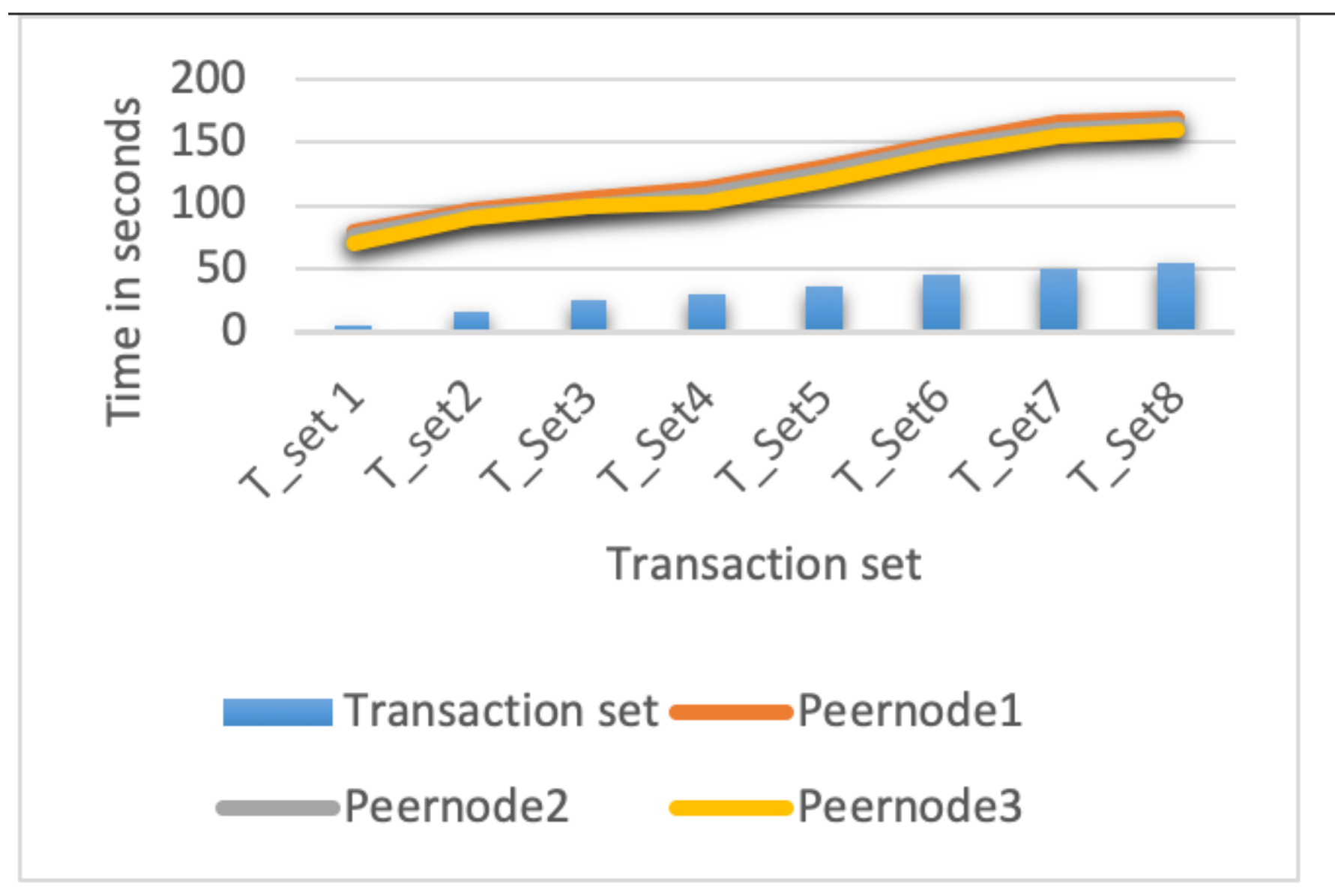

Figure 6

Latency comparison with organization 3 peer nodes taking sample set of transactions. 


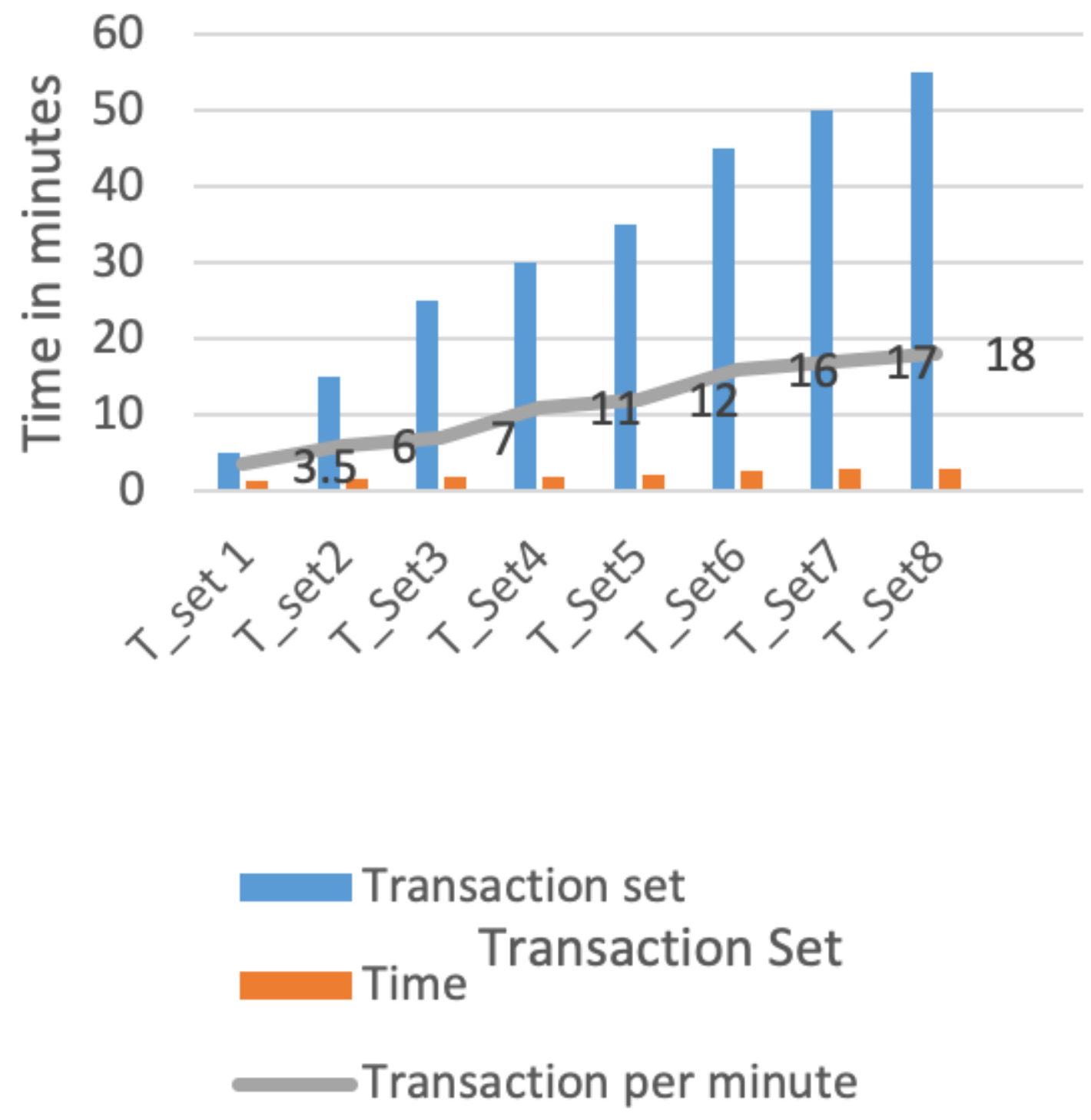

Figure 7

Throughput of Organization Peernode1 


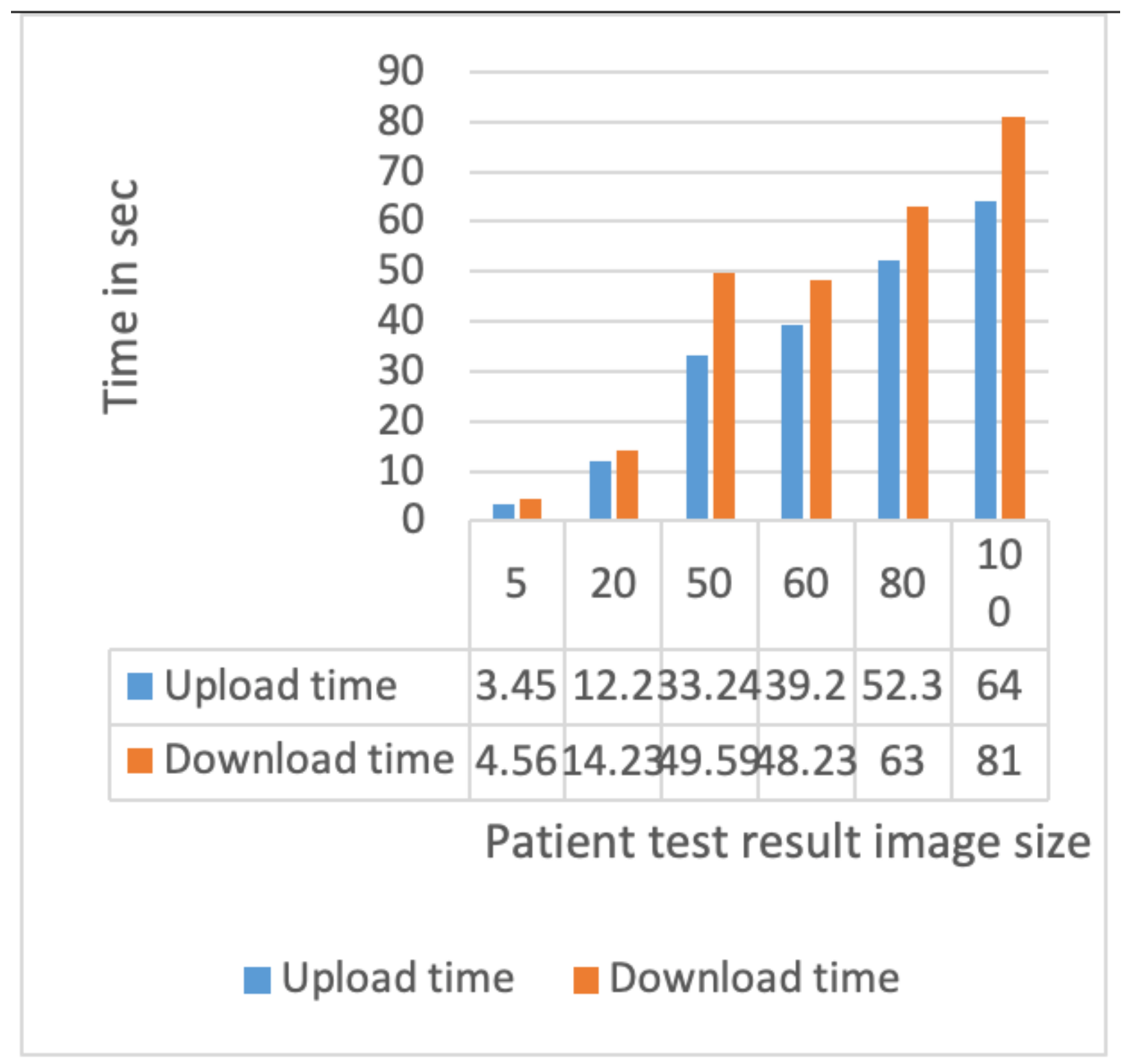

Figure 8

Average upload and download time of patient lab result as image using PCHDM 


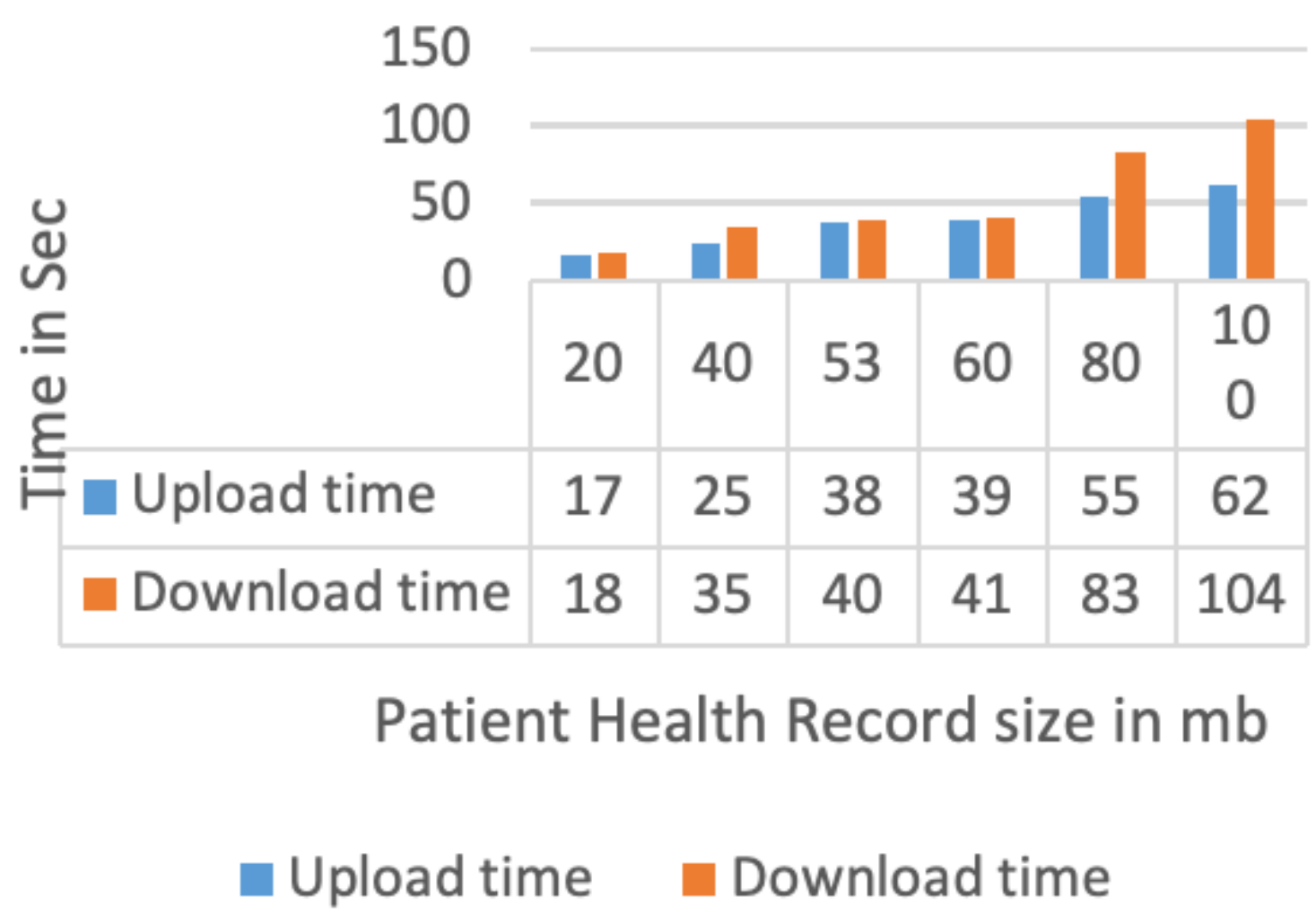

Figure 9

Average upload and download time of patient health record using PCHDM 


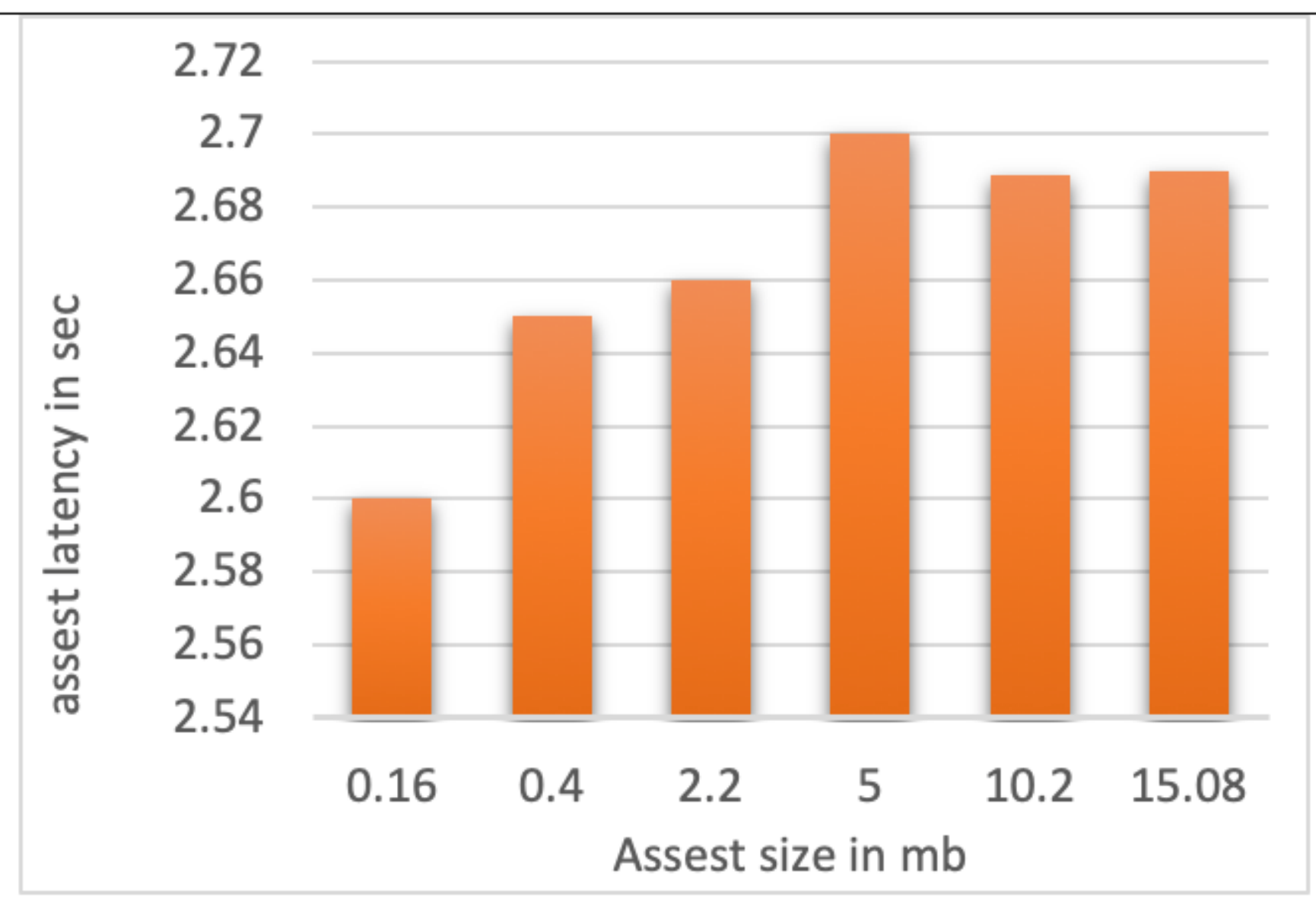

Figure 10

Average time taken for asset submission and response 